\title{
Progression of Focused Helium Ion Beam Milling in Gold Substrates
}

\author{
E.M. Mutunga ${ }^{1}$, S. Tan ${ }^{2}$, A.E. Vladár ${ }^{3}$, and K.L. Klein ${ }^{3,4^{*}}$ \\ 1. University of Tennessee, Knoxville, TN 37996 \\ 2. Intel Corporation, Santa Clara, CA 95054 \\ 3. National Institute of Standards and Technology, Gaithersburg, MD 20899 \\ 4. University of the District of Columbia, Washington, DC 20008 \\ *Corresponding author: kate.klein@udc.edu
}

The focused helium ion beam-solid state material interaction volumes differ in thin membranes and in bulk substrates $[1,2]$. While nanometer-scale structures with feature sizes of $5 \mathrm{~nm}$ or smaller have been machined in thin membranes [3-5], inconsistent results have been observed in bulk substrates where the irradiated region often swells rather than mills [6]. The depth and breadth of the interaction volume scales with beam energy, and the sputter yield also varies significantly. In this study, we seek to explore and understand these differences by observing the progression of focused helium ion beam milling. For this we used transmission electron microscope images of the beam-irradiated cross sections of 100-nm-thick gold membrane and 400-nm-thick (essentially bulk) gold substrates. We varied the ion beam landing energies and the dose as well.

Recent work [2] compared the interaction profiles of a $35 \mathrm{keV}$ helium ion beam of varied dose in 140nm-thick single crystalline silicon membrane and bulk samples using TEM cross-sectional imaging. It was found that forward sputtering is up to 5 times higher than backward sputter yields and is more significant in thin membranes where the thickness approaches the nuclear stopping range of the ions in the bulk substrate. Here we look at the machining properties of gold using similar techniques and further explore the effect of beam energy on this interaction profile. Line arrays (Figure 1) have been patterned using a $35 \mathrm{keV}$ helium ion beam in 100-nm-thick gold foil (a) and gold bulk substrate (b) using comparable dose. The gold bulk substrate thickness exceeds the ion range of $97 \mathrm{~nm}$ indicated by SRIM [7] helium ion point trajectories for a $35 \mathrm{keV}$ beam. This results in increased helium implantation in the material and causes more pronounced surface distortion compared to the $100 \mathrm{~nm}$ gold foil where approximately half of the ions are transmitted through the thin membrane. Nanomachining in bulk substrates is difficult due to this increased helium implantation in the material, which causes both surface and sub-surface damage. It is therefore desirable to find the optimal sputter yield conditions in addition to using the lowest dose feasible. Modeling results show higher sputter yields relate to lower beam energies. A graph of sputtering yields for various gold sample thicknesses at 1-50 keV beam energies is shown in Figure 2, where the theoretical sputter yield peaks between $5-7 \mathrm{keV}$. It was also experimentally observed that for $20 \mathrm{keV}$ as compared to $35 \mathrm{keV}$, the mill rate in a $100 \mathrm{~nm}$ gold foil increases by approximately $20 \%$.

[1] E. Mutunga et al, EIPBN Proceedings (2014).

[2] S. Tan et al, J. Vac. Sci. Technol. B 32, 06FA01 (2014)

[3] L. Scipioni et al, J. Vac. Sci. Technol. B 28, C6P18 (2010).

[4] J. Yang et al, Nanotechnology 22, 285310, (2011).

[5] L. Scipioni, Carl Zeiss, Adv. Mat. Char.Workshop, U. Illinois, (2012).

[6] R. Livengood et al, J. Vac. Sci. Technol. B 27 (2009) 3244.

[7] J.F. Zeigler, M.D. Zeigler and J.P. Biersack, SRIM-2012.03 modeling freeware. 


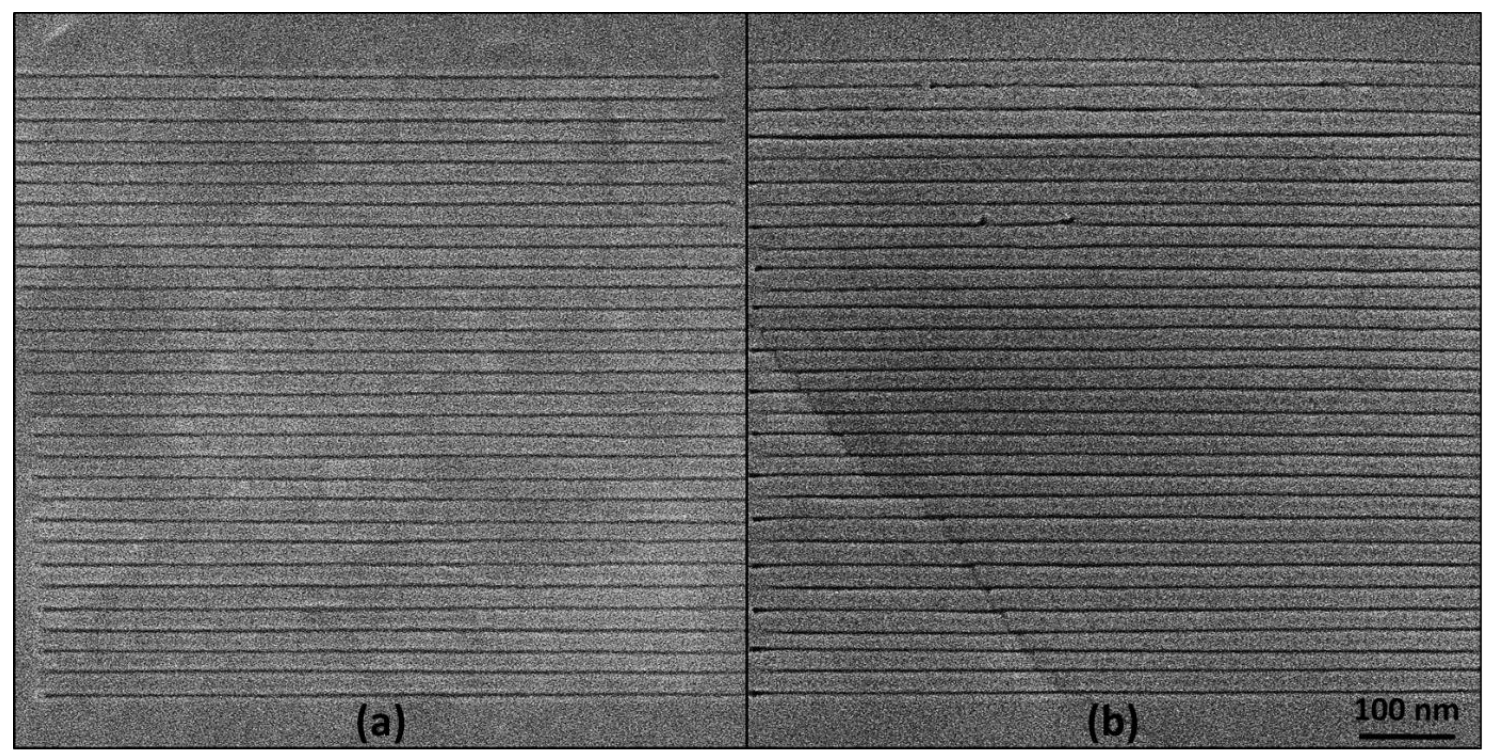

Figure 1. HIM micrographs of one-pixel-wide line arrays patterned at $30 \mathrm{~nm}$ pitch over a $1 \mu \mathrm{m}$ field-ofview using a $35 \mathrm{keV}$ helium ion beam on (a) 100-nm-thick gold foil and (b) 400-nm-thick gold bulk samples at a dose of $3.12 \times 10^{5}$ ions $/ \mathrm{nm}$. Surface distortion on the bulk sample is evident due to the accumulation of dislocations and damage caused by the trapped helium in the surrounding and underlying areas.

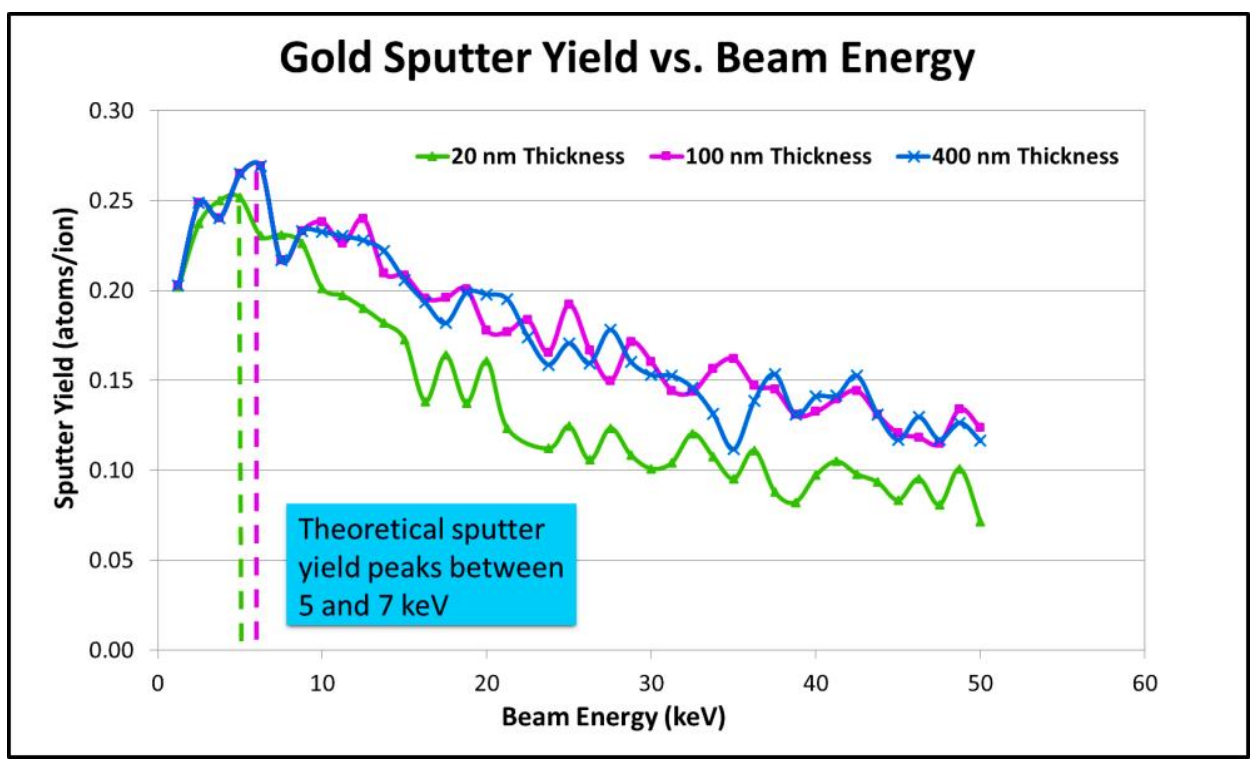

Figure 2. Plot showing theoretical backward sputter yield as a function of beam energy. Note the decreased sputter yield with increased beam energy for various thicknesses of gold. 